\title{
Elementos para soñar la Universidad ${ }^{1}$
}

\author{
Rolando Quesada Sancho*
}

\begin{abstract}
RESUMEN
Las discusiones que día con día se generan en los diferentes espacios académicos, tienen como referente el estado actual de la educación superior. De manera que las reflexiones y discusiones que se pueden elaborar acerca del estado actual y futuro de la educación superior y particularmente de la Universidad de Costa Rica son abundantes. Aquí se comparten algunas de las ideas, que emergen de la realidad universitaria. Se trata más que de exposición, de problematizar una situación cotidiana. En otras palabras, se trata de soñar con posibilidades.
\end{abstract}

Palabras clave:Prospección, Educación Superior, Universidad de Costa Rica, Cambio de época

\begin{abstract}
Discussions that everyday are generated through the different academic spaces, refer to the actual condition of the senior education. So, the reflections and discussions that can be made about the actual and future condition of senior education, and particularly from Universidad de Costa Rica, are abundant. Here are shared many of the ideas that emerge from the university's reality. It is more than an exposition, is to problematize an everyday situation. In other words, it is about dreaming with possibilities.
\end{abstract}

Keywords: Prospection, Senior Education, Universidad de Costa Rica, epoch's change

\section{INTRODUCCIÓN}

La educación superior pública y la universitaria particularmente, ha estado cuestionada desde hace ya décadas, por los sectores privados y gubernamentales. Mucha agua ha pasado bajo el puente, y son múltiples las hipótesis que han surgido de esa vorágine, que según los críticos afectan o inciden negativamente en la educación universitaria pública.

\footnotetext{
${ }^{1}$ Este ensayo tiene su sustento en reflexiones producidas durante investigación EDUCACIÓN SUPERIOR PÚBLICA COSTARRICENSE: ANÁLISIS PROSPECTIVO Y DESAFIOS DE LA UNIVERSIDAD DE COSTA RICA. INIE.

* Rolando Quesada Sancho. Costarricense. Antropólogo. Doctor en Educación. Profesor de la Escuela de Antropología, Universidad de Costa Rica, rolando.quesada@ucr.ac.cr
} 
Algunas de las respuestas o aproximaciones que se han externado, han ido conformando un imaginario colectivo multiforme y policromo, donde una idea que apenas llega con el fin de consolidarse, es desplazada por otra nueva, o resignificada y reinterpretada originando, en el mejor de los casos, un remozamiento de los marcos conceptuales vigentes. En otros se ha producido el enraizamiento de viejas estructuras y concepciones, mientras contingentes de personas desprecian otras ideas que las puedan separar de aquellas que ya habían adquirido y defienden sus posicionamientos formados durante su paso como estudiantes en las universidades, reduciendo los límites para los cambios. De esta forma nos encontramos con grupos que pretenden cambios profundos, otros prefieren cosméticos o bien aquellos que se estacionan en lo dado.

Es por eso que, en estas transformaciones, se debe contemplar la posibilidad de que, no todo cambio significa un avance en la reconstrucción de una universidad pública de cara a las necesidades sociales, por el contrario algunas de las novedades ideológicas pueden ir en detrimento de esa relación que es la que da sentido a las instituciones de educación pública.

De modo que en el espacio universitario nos encontramos con ese movimiento donde se antagonizan, niegan, priorizan, hibridizan ideas, muchas veces regidas por una estructura consolidada, otras veces construyendo nuevas, que van reconfigurando los modelos universitarios vigentes.

Externamos en este ensayo, algunas de las reflexiones generadas a partir de preocupaciones cotidianas, que con frecuencia se externan en los diferentes dependencias de la institución. En la exposición se teje un entramado de ideas que deberían de considerarse toda vez que pensemos la universidad, pues nos avecinamos a un cambio de época que demanda otros modelos de universidad. No pretendemos con ello dar soluciones a problemas existentes, sino de aportar elementos que ayuden a problematizar la realidad universitaria y con ello permitirnos soñar con una institución para el futuro. Por ello la discusión se orienta a ofrecer algunos elementos que nos estimulen romper los cercos del presente para mirar el horizonte.

\section{ELEMENTOS PARA UN CONTEXTO}

Los movimientos de cambio, no deben interpretarse como un evento intrauniversitario solamente, por el contrario, están atravesados por influencias externas al cerco universitario y por los intereses que se puedan generar desde los sectores productivos y dominantes del país y de los gobiernos nacionales. Es posible identificar orientaciones en la forma de conducir la institución y de "hacer" vida universitaria, que más adhieren a los intereses de algunos sectores y de los organismos multilaterales que a las necesidades sociales locales. Esto porque vivimos una inmediatez que tiende a reprimir algunos intentos de reflexión acerca de la vida universitaria desde una perspectiva histórica y de futuro.

Lo anterior obedece fundamentalmente al hecho de que a los organismos como el Fondo Mundial Internacional (FMI), Banco Interamericano para el Desarrollo (BID), Banco Mundial (BM), entre otros, adhieren también otros sectores y personajes, que desde el lugar que ocupan 
en la estructura social y cultural, coadyuvan a formar opinión, y crear semi verdades muchas veces asociadas a intereses particulares. De esa manera, los medios de comunicación, algunas personas dirigentes políticas, algunos gremios y asociaciones, el mercado laboral, entre otros, presionan para reorientar el modelo universitario con el fin de que ofrezca nuevas opciones al mercado con la lógica de la oferta y la demanda, independientemente de las necesidades que presenta la sociedad, confabulando de esa manera con la pertinencia de la formación superior.

La internacionalización de la información y del conocimiento, paulatinamente permea todas las instituciones educativas, entre ellas las universitarias, con diferentes ritmos y grados de participación en la asunción, producción, transferencia y difusión del saber científico, tecnológico y humanístico y va reconfigurando el sentido de la formación universitaria. Ella, se manifiesta en contenidos, metodologías, formas de organización, soportes tecnológicos y por supuesto las relaciones sociales, abriendo intersticios para modelar la universidad no solo en correspondencia con las necesidades sociales, sino muchas veces se prioriza el mercado nacional e internacional.

Estos elementos contextuales presionan a las instituciones para seguir reformándose paulatina e imperceptiblemente, promover reformas profundas y radicales o seguir estacionadas dando respuestas obsoletas a las nuevas necesidades sociales. Algunas de estas opciones o las tres simultáneamente, pueden experimentarse en algunas de las instituciones universitarias, producto de las relaciones que se crean entre los actores universitarios y el contexto.

La Universidad de Costa Rica, igual que otras instituciones homólogas, se configura como entramados de relaciones caracterizadas por pujas, cuya plataforma está dibujada por la misión descrita en el Estatuto Orgánico. Allí se puede leer un modelo de universidad que teóricamente daría no solo un referente para la construcción de una identidad universitaria, sino pautaría la ruta por la cual seguiría la institución. Sin embargo las múltiples relaciones y construcciones simbólicas que la modelan, han derivado en la constitución formal e informal, de modelos de universidad que hoy conviven y que surgen a partir de los movimientos de ideas antes comentadas.

Tal como dice Dávila

la estructura educativa no sólo tuvo ni tiene como función desplegar la esencia humana sino deteriorar algunas esferas de lo social y reforzar otras o contribuir a construir nuevas estructuras heterogéneas que, en cada momento y comparativamente con el estado del momento anterior, contribuyen al enriquecimiento o decrecimiento de los componentes esenciales (1996: 136).

De manera que pensar el estado actual de la Universidad de Costa Rica, nos obliga a reflexionar también acerca de su pasado y de sus procesos de cambio. Es allí donde se puede configurar la plataforma que permita mirar el horizonte para dibujar la universidad que necesitarán nuestras sociedades y que hoy podemos soñar. 
Pareciera entonces que nos encontramos en un cruce de vías que nos propone continuar con la inmediatez y seguir con reformas cotidianas sin una planificación adecuada o buscar una posibilidad prospectiva para una planificación pertinente. La reflexión siguiente trata de ofrecer algunos referentes que nos permitan abrir una discusión acerca de lo que queremos para la universidad pública costarricense y, específicamente para la Universidad de Costa Rica.

Poner en agenda de la población universitaria esta temática, abre portillos para preocuparnos por el modelo de institución que afronte las nuevas demandas sociales, culturales, económicas, políticas, entre otras, que se están presentando. De ahí que las preguntas ¿cuál es el modelo o modelos de universidad pertinente? y aunque parezca romántico ¿cómo es la universidad de mis sueños?, son medulares para iniciar una discusión en torno al cambio o reforma universitaria. Las múltiples respuestas que podrían arrojar estas interrogantes enriquecen aun más la discusión, pero simultáneamente nos permite construir en conjunto y con claridad esa institución necesaria y pertinente para las próximas décadas.

\section{ELEMENTOS QUE CONFORMAN PREOCUPACIONES COTIDIANAS}

Las discusiones espontáneas que día con día se generan en los diferentes espacios académicos, tienen como referente el estado actual de la educación superior. Académicos, académicas, estudiantes y personal administrativo, participan de tertulias donde se emiten supuestos, hipótesis y posibles respuestas a los problemas que, en tanto universitarios y universitarias perciben y por qué no, sufren. La observación y atención a dichas preocupaciones, están sustentando el interés por elaborar un estudio que dé cuenta de algunos tópicos que preocupan a la comunidad universitaria.

No son escasas las reflexiones y discusiones que se pueden elaborar acerca del estado actual y futuro de la educación superior, particularmente de la Universidad de Costa Rica. Las mismas son susceptibles de ubicarlas en fases de acuerdo con criterios tales como: temática, urgencia, viabilidad, competencia, entre otros. Aquí se comparten algunas de las ideas, que emergen de la realidad universitaria, su trayectoria y las tendencias actuales.

Posiblemente para nosotros, el supuesto más fuerte que subyace en muchas conversaciones y foros institucionales, es que la educación superior pública se ha transformado y continúa transformándose, sin una planificación adecuada y coherente con la historia y la experiencia presente de la realidad del país y de la región, así que las transformaciones la separan cada vez más de su misión originaria.

Las siguientes ideas, entonces, tratan de aglomerar y sintetizar a la vez, muchas de las reflexiones que cotidianamente se externan en la comunidad universitaria, ya sea como crítica, justificación, orientación, o deber, de la educación superior pública y de la Universidad de Costa Rica en particular, sin abordarse en forma sistemática y rigurosa.

Hemos tratado de resumirlas con el fin de dibujar el escenario que pisamos diariamente. 
- Se ha producido una ruptura del contrato fundacional, que delinea la relación entre la educación superior pública y la sociedad civil.

- El incremento desmedido y desordenado de las universidades privadas, con una lógica mercantil, han modificado la geografía universitaria del país. No existe una regulación adecuada y por el contrario la desregulación muchas veces actúa en la universidad pública.

- Existe una fuerte tendencia para que las universidades públicas satisfagan las necesidades del mercado, y se separen cada vez más de las necesidades de la sociedad. Un ejemplo muchas veces abordado es la venta de servicios descontrolada.

- Cada vez con más frecuencia, el gobierno solicita a las universidades públicas que encuentren formas de autofinanciarse, con lo cual se estimula la venta de servicios en forma desmedida.

- La ecuación reducción presupuestaria vis à vis el incremento de la población estudiantil, promueve la venta de servicios. La misma es estimulada por los salarios poco competitivos, con que permanecen los y las profesoras universitarias.

- Impera en las evaluaciones institucionales una tendencia a medir impacto, respondiendo nuevamente a las exigencias de entidades externas más que las internas. Lo procesal, que debe evaluarse cuando se trata de conocimiento se margina, opacando los logros en aspectos sustanciales para priorizar en lo observable y demostrable, como indicador de la calidad.

- La forma de contratación de personal docente e investigador, que rige no solo en la Universidad de Costa Rica, sino en otras universidades públicas, socava la actividad académica y coarta las posibilidades de comprometerse con la institución. Los nombramientos interinos promueven la inestabilidad, la desigualdad, y desestructuran las identidades universitarias y la fragmentación laboral.

- Han proliferado múltiples modelos de universidad que se expresan en las relaciones Universidad-Estado; Universidad-empresa; Universidad-sociedad civil.

- Es incipiente la discusión respecto al estado de la educación superior desde las instituciones, no coadyuva para la formación de una identidad universitaria, capaz de reivindicar algunas relaciones ya marchitas.

- Existe una clara tendencia a priorizar los procesos administrativos en detrimento de las actividades docentes, de investigación y de acción social. Esto deriva en sustentar un modelo tecnicista.

- En la Universidad de Costa Rica se ha privilegiado la investigación en las ciencias naturales, de la salud y, económicas, mientras que se han relegado otras áreas o disciplinas. De manera que este posicionamiento apoya la conformación de grupos al interior de la institución que posen mayores recursos para ejercer el poder. 
Cada una de estos enunciados y otros que no se incluyen, ameritan un trabajo de reflexión y síntesis que nos permita abrir nuevas rutas que orienten el ideal de universidad. No obstante, en este trabajo, cumplen con la función de recordar que para pensar en un modelo de universidad pertinente se debe conocer y dar respuesta a esos tópicos.

\section{ELEMENTOS PARA COMPRENDER LA INSTITUCIÓN UNIVERSITARIA}

Cuando pensamos en una institución educativa, debemos tomar en consideración que allí convergen una diversidad de referentes culturales provenientes de distintos actores sociales que participan del quehacer educativo, así como de referentes estructurales que modelan la institución. De manera que debemos tener clara la manera en que conceptuamos la institución universitaria, pues así, podemos trazar y orientar nuestros intereses personales e institucionales.

Podemos asegurar que la institución universitaria representa una urdimbre de relaciones sociales, que crean un campo de encuentros y desencuentros, en tanto que son construcciones que resultan de distintas coyunturas históricas y que entre otros objetivos, responden a las necesidades sociales. Toda institución se construye y mantiene la impronta de su contrato fundacional y es portadora a la vez de un mandato social.

La Universidad, en tanto institución educativa, tiene la especificidad pedagógica, y nuclea ${ }^{2}$ una diversidad de actividades, donde se elaboran las relaciones entre la persona y su entorno sociocultural. Tales relaciones, pendulan entre dos tendencias, una reproductivista y la otra innovadora, generadora de los satisfactores de las necesidades sociales en momentos históricos específicos.

Cuando hablamos de una institución educativa, nos referimos pues, aquel espacio donde se realizan acciones o prácticas, se construyen teorías, se norman relaciones, se resignifican saberes, costumbres, ritos, creencias, entre otros, para poner en contacto a profesionales y sus saberes científicos dirigidos a una población específica, en otras palabras se construyen modelos.

De acuerdo con Beltrán

la institución se nos muestra, desde sus elementos de tensión, como un espacio no sólo dotado de dinámica interna, sino también externa; un espacio en el que operan confrontación y lucha, cambio y redefinición de las reglas que son quienes le confieren su aparente "entidad” bajo una visión diacrónica. (1997: 69).

Empero la dinámica que experimenta una universidad no es la consecuencia únicamente de la voluntad o interés de las y los actores sino que intervienen las políticas y las reglas que los y las afectan en los procesos educativos. Sin embargo no debemos pretender comprender los cambios institucionales como alteraciones de la aplicación de políticas, como tampoco con la sola mirada

\footnotetext{
${ }^{2}$ En este trabajo se utiliza el verbo nuclear según la usanza Argentina, el cual tiene la acepción de agrupar, (cf: diccionario "Pequeño Larousse Ilustrado (1992). Bogotá: Ediciones Larousse).
} 
al quehacer de las y los actores quienes pueden, a título personal, provocar cambios. Por el contrario se trata de sustentar el cambio en las continuidades y rupturas, que constituyen las características fundamentales de la institución, desde este lugar el cambio aparece como un proceso simultáneo de reestructuración y reinterpretación.

La universidad, en tanto institución educativa, cumple la función de orden y control social, esto porque en esos espacios los actores actúan con base en la manera en que las normas los enajenan y en como aportan en las construcciones simbólicas, formando así consenso ideológico y cultural o de socialización mediante la internalización de esquemas de valores y de normas legitimadas socialmente (Tünnermann, 1990).

Sin embargo, la estructura educativa puede privilegiar mecanismos y técnicas estereotipadas, procesos de mera transmisión o inculcación de conductas, aprendizajes y adiestramientos instrumentalistas, resultando una estructura social y cultural estática. Por el contrario, si enfatiza en procesos no habituales, no rutinarios y no estereotipados, estaría aportando a la innovación y al cambio, en ese sentido estimularía el dinamismo de las estructuras.

Algunas de estas estructuras son una constante construcción y reconstrucción de un orden, donde las y los actores participantes lo hacen en correspondencia con su reservorio material y simbólico que han adquirido en su experiencia de vida. En esta constante interacción las personas convierten su participación en una acción política, de tal manera que el estudio de la institución debe centrarse precisamente allí, en los procesos de organización pues en ellos se restauran, se construyen o se reprueban las reglas que son las que estabilizan y dan permanencia a la institución, resguardando en su interior el proceso de reinstitucionalización.

La estructura universitaria puede fungir como medio para la transmisión de nuevos significantes. Pero la función de la estructura educativa no acaba con la transmisión, sino que es creadora, constructora de nuevos conocimientos, evaluadora y crítica de los antiguos o nuevos significados y, es generadora de comportamientos y valores. Esto revalora el conocimiento en sus dos modalidades: conocimiento común y conocimiento científico.

Cuando una sociedad está en crisis, afloran varias esferas de significación que pugnan por ganar primacía social, lo que provoca nuevas formas de organizarse y de desarrollarse el conocimiento, así como nuevos valores y pautas de comportamiento (Beltrán, 1997).

Indudablemente la universidad es una institución, cultural y científica por excelencia y constituye uno de los aportes sobresalientes del mundo occidental (Tünnermann, 1990). Con su instalación, era posible sistematizar y difundir el conocimiento, proveer a la sociedad civil de otros recursos para afrontar la cotidianeidad, así se puede hablar del desarrollo técnico científico logrado por la sociedad de la época, que fue incentivado por la acción de las universidades. Estas se convirtieron en las formadoras del semillero de los gobernantes de los países del primer mundo, función que algunas se resisten a abandonar. 
No debemos pensar las Universidades como un espacio de consenso de ideas solamente, sino como un lugar donde la polémica y la discusión de temas que eventualmente pueden "herir susceptibilidades" provocan reacciones adversas.

Desde sus orígenes y conforme el conocimiento se desarrollaba surgían pugnas entre los grupos que detentaban el poder político y religioso, por las nuevas ideas y saberes que se desarrollaban, y que deslegitimaban el "conocimiento absoluto" que ellos defendían y difundían para perpetuarse en los poderes. No fueron pocos los pensadores que tuvieron que abandonar las universidades víctimas de persecuciones. Esta situación pareciera que no ha terminado de repetirse, en tanto que el conocimiento plantea la pluralidad como condición propia para su reelaboración, lo que deviene en deslegitimación y desautorización de formas de ejercer el poder.

La Universidad es un entramado de relaciones de diverso tipo, constituido "por los agentes sociales que intervienen para que un tipo particular de producto se haga, circule, se consuma en la sociedad, tiene leyes propias, participa de la totalidad de la estructura social, a la vez, está regido por un funcionamiento específico". (García, 1995: 30)

Este entramado configura su propia estructura, su propio modelo, así cada participante posee recursos propios innatos o aprehendidos, que le permite relacionarse en forma asimétrica, $\mathrm{y}$ posicionarse de un estatus para establecer negociaciones en ese terreno cuyo límite queda determinado por la finalización del efecto de campo. Cuatro son las formas de recursos o capital que reconoce Pierre Bourdieu y que cada participante puede movilizar para conseguir su finalidad: económico (recursos monetario), cultural (referido a herencia familiar o educativa), social (conexiones y membresía a un grupo) y simbólico (forma que adquieren los diversos tipos de capital cuando son asumidos); (Auyero, 1996).

Algunos rasgos propios de la Universidad, lleva a pensar que la contradicción con lo establecido le fue propia en determinadas épocas. Los hábitos intelectuales y morales y el espíritu crítico que se fomenta desde esta institución convoca al interrogatorio de lo estatuido, configurándose como crisol de ideas, pensamientos, desarrollos teóricos, entre otros.

Se reconoce también, que el conocimiento es la columna vertebral de los sistemas de educación superior aunado con los sujetos que lo reconstruyen y lo transmiten y "se compone de múltiples células de especialización colocadas horizontalmente y débilmente articuladas en los niveles de coordinación" (Clark, 1983: 41).

Las universidades tienen objetivos que trascienden a las coyunturas de espacio, tiempo y recursos, y a las propias desigualdades geográficas y sociales. Pero sí deben responder a un momento histórico determinado, su vinculación con la sociedad debe estar permanentemente en reconstrucción, debe estar en correspondencia con el desarrollo tecnológico, económico, político y cultural. Por lo tanto, se puede inferir que es importante que sus intereses se orienten a contribuir con el desarrollo integral articulando las ciencias, las humanidades y las artes para aprehender la realidad nacional. Es necesaria la producción de una "cultura" general que permita la criticidad, para la convivencia en libertad y por otro lado la reconstrucción de conocimiento y tecnología necesaria para el desarrollo económico de los países. 
En países pobres las universidades deben de imprimirle a su labor académica un sentido realmente útil, convocar a sus estudiantes para que se comprometan en los procesos de transformación de las sociedades, para tratar de mejorar las condiciones de existencia de la mayoría de sus pobladores, de esta manera la universidad se integra a los procesos de cambio, no como un ente suspendido sino como una instancia de la población civil, para lo cual es condición sine qua non que la universidad sea libre.

La comprensión de la Universidad desde esta complejidad, puede significar un reto para aceptar que lejos de seguir proponiendo reformas cotidianas con un sentido instrumental, se trata de planificarlas desde los contextos globales, locales e institucionales.

\section{ELEMENTOS PARA PENSAR PROSPECTIVAMENTE}

Lo dicho en los apartados precedentes, nos permite asegurar que la educación superior conforma una pieza fundamental en el desarrollo económico, social, cultural y político del país y, los grupos humanos que participan de y en ella, son co-responsables de la construcción del presente y del futuro de los países. Estos procesos son susceptibles de influenciarse por las ideologías dominantes, de modo que en su interior tienden a reproducirse y consolidarse. Esto conforma una arena movediza donde los grupos minoritarios tenderán a entrar en conflicto para legitimar su voz.

El cúmulo de transformaciones parece advertir que va más allá de una época de cambios, por el contrario -tal como nos dice De Souza (2001)- se trata de un cambio de época. La primera se caracteriza porque su identidad está establecida en forma inequívoca, y sus características son reconocidas sin ser cuestionadas en sus consecuencias y la segunda es un momento de la historia de la humanidad donde las características están en deterioro irreversible y sus consecuencias para el desarrollo están bajo cuestionamiento inexorable por parte de la mayoría de la sociedad.

De Souza, conjuntamente con Castells, afirma que "un nuevo mundo está emergiendo a partir de la coincidencia histórica entre tres procesos que, de forma independiente, se iniciaron alrededor de finales de los 60 e inicios de los 70" (2001). Estos procesos son: la revolución en torno a la tecnología de la información; la crisis económica del capitalismo y estatismo; y la explosión planetaria de movimientos sociales y culturales (feminismo, liberación sexual, derechos humanos, ambientalismo).

Estos procesos, según el autor citado, están transformando en forma diferenciada en su capacidad de impacto, simultáneamente las estructuras de las relaciones de producción, de poder y la cultura, estructuras que son y configuran las sociedades; en su lugar se constituyen redes que son las que tienden a moldear la sociedad (De Souza, 2001).

Algunos de los procesos productivos ingresan en el campo de la obsolescencia, mientras emergen otros como el conocimiento, que es aplicado sobre conocimiento, para producir más conocimiento que tiende a transformarse en mercancía, así el mismo es insumo y producto a la vez. Esto hace bisagra con la transformación de las relaciones de poder, producto de los acuerdos 
multilaterales. Los actores globales con más poder deciden o influencian las decisiones de los estados-nación, de manera que autoridad y legitimidad se ven seriamente erosionadas. Los estados-nación pierden así su espacio, y en su lugar se asienta la corporación transnacional, la cual en tanto forma de acumulación de poder material y simbólico, se distingue por la insensibilidad a la desigualdad social.

El espacio tiempo, es otro factor que se altera con los avances tecnológicos, emerge con ello prácticas culturales asociadas a la realidad virtual, que vertiginosamente se apropia de cuanto reducto encuentre fértil, de esa manera de aquí en adelante la realidad es aquella que presentan los medios, que logran empoderarse cada vez más, cuanto lo virtual contradictoriamente se consolida (De Souza, 2001).

Dada estas condiciones, no debemos seguir transitando sin esperanza, pues ella es la constructora de horizontes que son los que proveen de contenidos renovadores para resignificar las realidades. Esos horizontes son la aventura de asomarse a lo inédito y por lo tanto incierto, que se caracteriza por su pluralidad de posibilidades y por la ruptura con lo dado.

Pensar los horizontes, también significa traspasar el límite y mirar aquellos que contiene en su interior, pero a la vez aquello que lo trasciende que no es más que la génesis del horizonte, situación que contiene la capacidad de producir asombro. "De ahí que el conocimiento es el asombro vuelto sobre sí mismo, mientras que el asombro es el conocimiento como capacidad de salirse de sí mismo para rastrear las incertidumbres y encontrar lo maravilloso vislumbrado para traerlo a la quietud de su constatación". (Zemelman, 1992b: 167)

Es necesario instalar en las universidades un discurso que asuma en forma cotidiana la responsabilidad intelectual y ética de la gestación de realidades que se caractericen por su capacidad para articular contenidos teóricos con horizontes históricos y sus múltiples opciones de construcción.

En ese sentido "la historia del hombre enseña acerca de los esfuerzos por liberarse de estas trabas del pensamiento y de la imaginación, entre los que cabe destacar, por su libertad y profunda capacidad de anticipación en comparación con la misma ciencia, aquellos que provienen del arte" (Zemelman, 1992b: 170). Y por tanto promueven la trasgresión de los umbrales propuestos por la cultura de referencia u oficialista, para proponer nuevas alternativas. Estas rupturas plantean la construcción de una conciencia crítica.

Es así que el rescate de los actores sociales, hombres y mujeres, se realiza desde la concepción del sujeto en su historia, en tanto que lo coloca en la posibilidad de construcción de nuevas oportunidades. Asumido como sujeto histórico, lo reducimos a la determinación. Se puede derivar de aquí que la historia es experiencia y conciencia de horizontes, pues el sujeto trasciende el umbral de lo establecido, camina más allá del borde que lo limita y con base en la esperanza y el asombro, construye. Se coloca de esa manera entre el pasado y el futuro

Sin embargo, no podemos obviar que obstáculos varios llegarán al encuentro, en tanto que existe un entramado conceptual y cultural que contiene a la mente y la puede mantener en un estado 
perezoso, con lo cual nos corremos el riesgo de quedarnos con dado, respondiendo muchas veces al conservadurismo. Recordemos que nos desenvolvemos en un espacio altamente complejo, donde confluyen intereses diversos, tanto personales como institucionales y de los diferentes contextos.

La educación superior, no puede darle la espalda a éstos acontecimientos, de ahí que se ha advertido en múltiples ocasiones, que está siendo revalorada, dada la importancia que está adquiriendo tanto la información como el conocimiento. Esto porque una sociedad que transita hacia una fase que se fundamente en el conocimiento, tiene otras expectativas y demanda nuevas metas de la educación en general y terciaria en particular.

De modo que "La educación superior del futuro será una puerta de acceso a la sociedad del conocimiento, quizá la puerta más importante por su situación privilegiada para la generación y transmisión del saber humano" (ANUIES, 1998).

Si asumimos que estamos introduciéndonos en un cambio de época, donde la sociedad se transforma, pasando de la sociedad de la información a la sociedad del conocimiento, las universidades tradicionales coexistirán con otras que apenas llegan al escenario educativo, como es la universidad virtual, la universidad corporativa, u otras formas de estructura universitaria que hoy apenas se vislumbran.

Hay que reconocer que la sociedad del conocimiento, tiene su fundamento en la flexibilidad y en formas democráticas de convivencia, es por eso que los actores sociales deben tener una formación crítica y deben estar informados, con posibilidades de externar y compartir libremente sus ideas, de manera que la universidad debe responder a esa nueva realidad, ofreciendo una formación en aquellos valores acordes con la sociedad de la cual se es parte, con un sentido de futuro social basado en la democracia, la libertad y la justicia social.

No en vano, parte de la comunidad académica comparten que la Universidad "precisa de unos nuevos marcos que le permita autoorganizarse para responder a las necesidades y a los cambios en las diferentes esferas políticas, sociales, económicas, laborales y tecnológicas" y de esa manera "debería asumir aquel papel de liderazgo intelectual, crítico, científico que en diversos periodos históricos ha asumido", y continúan diciendo que "La universidad debe volver a responsabilizarse de la sociedad de la que forma parte y asumir el protagonismo como motor cultural y social que le confiere el privilegio de sus características". (Benedito, Ferrer y Ferreres, 1995: 33)

Reafirma lo anterior, el chileno Zemelman

lo desconocido no asume la forma de un enigma, sino que constituye un imperativo gnoseológico y volitivo para construir lo inédito. Ello supone no aceptar quedarse dentro de la estabilidad guarnecida por los límites; por el contrario, estamos obligados a embestirla en la búsqueda de una utopía como es la de la lucha por una conciencia protagónica, y así poder avanzar para 
enriquecer nuestra propia relación con la realidad, aprobada como ese horizonte que desafía como nuevo objeto a la voluntad consciente. (1992b: 166)

No podemos entonces permanecer separados de estas nuevas corrientes que dinamizan no solo la educación sino la sociedad en general. Específicamente lo referente a la universidad, pareciera que se debe encauzar a la búsqueda de otros modelos, y dejar atrás aquellos, hasta ahora sustentados por el Estado, en tanto que tienden a ser insuficientes.

En ese sentido pareciera que los modelos que se constituyeron en referente, para consolidar las instituciones universitarias, ya no son suficientes. El modelo humboldtiano y napoleónico, el primero como proyecto del saber por el saber, saber reconstituyente del Estado y la escuela especializada propio del napoleónico, y las múltiples formas de hibridación que han resultado de ambos, con pretensiones de encontrar un equilibrio entre los fines de uno con los del otro, "cuesta mantenerlo y entonces se decantan las universidades o bien por la formación general desde carreras a veces obsoletas o bien por la especialización y profesionalización exenta de formación humanística, intelectual y en valores”. (Benedito, Ferrer y Ferreres, 1995: 29)

Otros modelos que se configuran, a partir de las reformas instrumentales y de la constante y poco planificada reforma universitaria son: la universidad adaptadora, que apenas tiende a adaptarse a los cambios tecnológicos, científicos y en general a adaptarse a las demandas sociales, del mercado y laborales sin un referente consolidado; la universidad reguladora del mercado, que es aquella que se anticipa a las demandas y necesidades sociales, productivas y técnicas estableciendo una relación más recíproca con estas instancias; la universidad masificada, configurada a partir del fenómeno de masificación debido al desajuste entre la demanda y la oferta universitaria, generada durante los decenios del 70 y 80, con severas consecuencias en cuanto a atender un numeroso cuerpo de estudiantes en óptimas condiciones.

Otros elementos, que simultáneamente al desgaste de los modelos vigentes, hay que considerar en forma exigida, son aquellos, asociados con el abandono de las categorías únicas para pensar el mundo, esa racionalidad absoluta que muchas veces orientó el quehacer del y de la ciudadana del siglo recién pasado, parece que ha entrado en franca devaluación. Los grupos organizados (pacifistas, ecologistas, feministas y gays, entre otros) abogan por la interpretación de las realidades desde una perspectiva plural, con equidad e igualdad de oportunidades en el acceso a los bienes materiales y simbólicos. Esta crisis de la racionalidad cuestiona indudablemente el rol de la "universidad como regente -universal- del conocimiento, el saber, la técnica y las artes." (Benedito, Ferrer y Ferreres, 1995: 32)

Según Tedesco (2000), el problema de acceso al conocimiento debería tomar en consideración al menos lo siguiente: que para responder a la nueva configuración social, es necesario educarse durante toda la vida, que la democratización del acceso a los niveles más complejos del conocimiento no puede quedar confinada al acceso a la universidad y que el acceso al conocimiento supone encarar los desafíos que plantean las nuevas tecnologías de la información a las instituciones y a los métodos de enseñanza. 
De manera que un país como Costa Rica, que en las últimas décadas ha apostado al turismo y a los capitales "golondrina", donde la inmigración ha reconfigurado la composición social y cultural, y se acentúa la exclusión social y el interés por expulsar del escenario nacional lo poco que queda del Estado de Bienestar vía un tratado de libre comercio, negociado con una clara posición desventajosa para nuestro país, se establecen pautas del estado actual y futuro cercano de las condiciones de vida que experimentaremos, mismas pautas deben ser criterios para revisitar la Educación Superior Pública en general y la universitaria en particular.

La universidad soñada debe buscar la pertinencia de acuerdo con su historia, y debe sentido lograr un sentido de pertenencia, con la finalidad de que la población universitaria, desarrolle las competencias para autorrevisarse y conocer sus fortalezas y debilidades para desarrollar una actitud, para lo cual debe revisitar la institución desde la complejidad, a partir de lo que cotidianamente se experimenta. Así podemos aproximarnos a la planificación de la universidad del futuro, tomando como referente la sociedad del conocimiento.

\section{ELEMENTOS PARA CONCLUIR}

Repensar el fin de la educación superior dentro de los contextos sociales, políticos, culturales, económicos parece ser la llave que nos pueda llevar a dibujar nuevos escenarios. Pero los escenarios no son plataformas vacías, son espacios donde confluyen entre otros elementos, la escenografía, el libreto, el vestuario y la música. Lo que obliga pensar a su vez, en la eventual acción, que protagonizan y antagonizan aquellos actores sociales que se encuentran en escena, y en el público para quien va dirigido el espectáculo.

De la misma manera, los escenarios no son cosas terminadas, sino determinaciones inacabadas, abierta a nuevas realidades, susceptible de recrear las determinaciones establecidas. Para seguir con la metáfora, los escenarios son realidades en movimiento, donde eventualmente actores, libreto, escenografía, música, pueden estar variando en relación con los contextos más amplios. Estos movimientos, sin embargo, no son solo el resultado de ciertos dinamismos con ciertas regularidades, sino que se refieren a movimientos susceptibles de ser construidos desde el presente, así un escenario potencial es desde el presente una realidad construible.

También, el presente mismo es para los actores sociales la manera en que se evidencia mediante la praxis, la apropiación que han realizado de los diferentes momentos vividos, $\mathrm{y}$ simultáneamente la construcción de la direccionalidad de ese presente hacia un futuro cercano.

Asumir el presente como momento dinámico, producto de un pasado susceptible de haber sido construido, lleva a pensar la historia como una secuencia de coyunturas, en donde se van a encontrar a los sujetos sociales actuando.

Desde luego esto nos propone un correlato que solicita el regreso de la interdisciplinariedad, como fundamento para mirar el futuro universitario y abordar las realidades que se avecinan, en donde el componente humanista estaría atravesando toda propuesta curricular. 
Finalmente se puede agregar que el futuro de la Universidad la pensamos con una propuesta curricular nueva que incluye las tecnologías de punta, con un fuerte acento en la educación continua y a distancia. Dicha propuesta estaría modificando a su vez la estructura administrativa vigente con lo cual se libera de su corsé a la gorda, esa entidad donde confluyen los procesos administrativos que, según el imaginario social, retrasan las propuestas académicas.

\section{BIBLIOGRAFÍA}

Anuies. (s.f.). Educación Superior en el Siglo XXI. Líneas estratégicas de desarrollo. Una propuesta de Anuies, México.

Beltrán, F. (1997). Las instituciones en el cruce de caminos. En: Frigerio, G.; M. Poggi y M. Giannnoni (Comp.). Política, instituciones y actores en educación. Argentina: Centro de estudios multidisciplinarios; Ediciones Novedades Educativas.

Benedito, V.; Ferrer, V.; Ferreres, V. (1995), La formación universitaria a debate. España: Publicacions de la Universitat de Barcelona.

Bourdieu, P.; Wacquant L.J.D. (1995), Respuestas por una antropología reflexiva. México. Editorial Grijalbo S.A.

Brovetto, J.; Juri, H.; Rojas, M.; Yarzábal, L. (1999). Reflexiones previas. En: Brovetto, J.; Juri, H.; Rojas, M.; Yarzábal, L: Universidad Iberoamericana. Globalización e Identidad. España: CEXECI Centro Extremeño de Estudios y Cooperación con Iberoamérica. AUGM Asociación de Universidades Grupo Montevideo.

Clark, B. (1983). El sistema de Educación Superior una visión comparativa de la organización académica. México: Universidad Autónoma Metropolitana-Azcapotzalco. Editorial Patria, S.A.

Dávila, F. (1996). Apuntes analíticos para la comprensión de la estructura educativa. En: de Alba, A. (Coord.). Teoría y educación en torno al carácter científico de la Educación. México: Universidad Autónoma de México.

De Souza, J. (2001). La dimensión institucional del desarrollo sostenible. De las "reglas de la vulnerabilidad" a las "premisas de la sostenibilidad" en el contexto del cambio de época. Costa Rica. (mimeo).

Didrickson, A. (2000). La universidad del futuro. México: Centro de Estudios sobre la Universidad. Plaza y Valdés Editores. Universidad Nacional Autónoma de México.

-----. (2002). Tendencias en la educación superior en el Mundo. En: Comboni, Juárez y París (coord) ¿Hacia dónde va la Universidad Pública? México: Universidad Autónoma Metropolitana, Unidad Xochimilco. 
Gacel-Ávila, J. (2003). La internacionalización de la educación superior. Paradigma para la ciudadanía global. México: Universidad de Guadalajara.

Gurdián, A. (1994). El curriculum universitario y los desafíos del futuro. En: Gurdián, A. (Comp.) Proyecto de universidad para el siglo XXI. Costa Rica: Oficina de publicaciones de la Universidad de Costa Rica.

----. (2000). Dinámica de la Educación Superior en Costa Rica: Desafíos y tendencias (19961999. (mimeo).

Jensen, H. (1995). Universidad ciencia y humanismo. Costa Rica: Editorial Tecnociencia.

Juárez J.M.; Comboni, S. (2002). Prólogo. En: Comboni, Juárez y París (coord) ¿Hacia dónde va la Universidad Pública? México: Universidad Autónoma Metropolitana, Unidad Xochimilco.

Levy, D. (1995). La educación superior y el Estado en Latinoamérica. Desafíos privados al predominio público. México: Centro de Estudios sobre la Universidad, UNAM. Facultad Latinoamericana de Ciencias Sociales, Sede México. Miguel Ángel Porrúa, Grupo Editorial.

López, F. (1999). Globalización y diversidad cultural En: Brovetto, J.; Juri, H.; Rojas, M.; Yarzábal, L. Universidad Iberoamericana. Globalización e Identidad. España: CEXECI Centro Extremeño de Estudios y Cooperación con Iberoamérica. AUGM Asociación de Universidades Grupo Montevideo.

López, O. (2004). Universidad del siglo XXI. San José: Editorial Guayacán.

Morin, E. (2002). Los siete saberes necesarios a la educación del futuro. Buenos Aires: Nueva Visión.

París, M.D. (2202). Introducción. En: Comboni, Juárez y París (coord) ¿Hacia dónde va la Universidad Pública? México: Universidad Autónoma Metropolitana, Unidad Xochimilco.

Pérez, A. (1998). Políticas del conocimiento: educación superior y universidad. Argentina: Editorial Biblos.

Quesada, J.R. (2003). La educación en Costa Rica: 1920-1949. En: Salazar, J.M. Historia de la educación costarricense”. Costa Rica: Editorial Universidad Estatal a Distancia, Editorial Universidad de Costa Rica.

Quesada, R. (2000). Cultura, poder y curriculum, todos en el mismo crisol. Educación. Revista de la Universidad de Costa Rica. Vol. 24(2).

-----. (2001a). Aproximaciones para llegar al fondo del "pozo". Evaluación curricular en los "cercos académicos". Revista de Ciencias Sociales. No. 93-94(II-III). 
-----. (2001b). Reforma curricular: rito y acto de fe. Revista de Ciencias Sociales. No. 94(IV).

Rojas, L. (1999). La función del intelectual de la Universidad: ¿una responsabilidad abandonada? En: Brovetto, J.; Juri, H.; Rojas, M.; Yarzábal, L. Universidad Iberoamericana. Globalización e Identidad. España: CEXECI Centro Extremeño de Estudios y Cooperación con Iberoamérica. AUGM Asociación de Universidades Grupo Montevideo.

Shwartzman, S. (1993). Políticas de Educación Superior en América Latina: el contexto. En: Políticas Comparadas de Educación Superior en América Latina. Chile: FLACSO-Chile.

Tedesco, J.C. (2000). Universidad y sociedad del conocimiento. En Gestión, evaluación y acreditación de instituciones de Educación Superior. Seminario regional (s.n.p.). Buenos Aires: IESALC. FLACSO-Argentina. OEI. IIEP.

Tünnermann, C. (1990). Ensayos sobre la teoría de la Universidad. Managua: Editorial Vanguardia.

-----. (1999). Educación Superior de cara al siglo XXI. Costa Rica: Sede de Occidente. Editorial Mirambel, S.A.

Universidad de Costa Rica. (1990). Estatuto Orgánico. Oficina de Publicaciones de la U.C.R.

Villaseñor, G. (1994). La universidad pública alternativa. México: Universidad Autónoma Metropolitana. Centro de Estudios Educativos, A.C.

Zemelman, H. (1992a). Los horizontes de la razón. I Dialéctica y apropiación del presente. España: Editorial Antropos.

----. (1992b). Los horizontes de la razón. II Historia y necesidad de utopía. España: Editorial Antropos. 